Communications in Physics, Vol. 22, No. 2 (2012), pp. 141-146

\title{
CURIE TEMPERATURE OF DILUTED MAGNETIC SEMICONDUCTORS: THE INFLUENCE OF THE ANTIFERROMAGNETIC EXCHANGE INTERACTION
}

\author{
VU KIM THAI \\ Institute of Physics, VAST \\ LE DUC ANH \\ Hanoi National University of Education \\ HOANG ANH TUAN \\ Institute of Physics, VAST
}

\begin{abstract}
The coherent potential approximation and mean field approximation are used to calculate the free energy of the coupled carrier localized spin system in III- $V$ diluted magnetic semiconductors. Thus the magnetic transition temperature $T_{c}$ can be determined and its dependence on important model parameters. We show that the strong antiferromagnetic superexchange interaction between nearest neighbour sites considerably reduces the Curie temperature.
\end{abstract}

\section{INTRODUCTION}

Diluted magnetic semiconductors (DMS) are semiconducting alloys where lattice is partly made up of substitutional magnetic atoms. The most extensively studied DMS in recent years are (III,Mn)V-type DMS, in which a fraction of the group III sublattice is replaced at random by magnetic Mn atom [1]. It is highly noteworthy that the doping of Mn into GaAs and InAs lead to ferromagnetism and magnetooptical and magnetotransport phenomena. So far, over the last ten years (Ga,Mn)As and related compounds have considerably strengthen their position as an outstanding playground to develop and test novel functionalities unique to a combination of ferromagnetic and semiconductor system. Many concepts, like spin-injection, electric-field control of the $T_{c}$ magnitude and magnetization direction, are being now developed in devices involving ferromagnetic metals, which may function at ambient temperatures. Therefore, a further increase of $T_{c}$, over current record value of $190 \mathrm{~K}$, continues to be a major goal in the field of DMS [2-3].

From theoretical point of view there are mainly two types of disorder in DMS: substitutional disorder and the thermal fluctuation of localized spins. Neglecting disorder effect the mean field Zener model predicts the possibility of high Curie temperature for some materials [4-5]. However, properly taking the disorder effect into consideration, as shown in some latter studies, is indispensable in calculation of the $T_{c}$ in DMS [6-8]. In almost theoretical works above, as far as we know, the influence of the direct exchange interaction between magnetic impurities has been neglected. The purpose of this paper is to calculate the magnetic transition temperature in III-V-type DMS where both of 
the exchange interaction between carrier and impurity spins, and the direct exchange interaction between magnetic impurities are taken into account. We show that the strong antiferromagnetic superexchange interaction between nearest neighbour sites considerably reduces the Curie temperature.

\section{THE MODEL AND FORMALISM}

In order to study the influence of the antiferromagnetic exchange interaction between magnetic impurities on the Curie temperature in DMS of the type $\mathrm{A}_{1-x} \mathrm{Mn}_{x} \mathrm{~B}$, where the parent material $\mathrm{AB}$ is assumed to be a nonmagnetic III-V compound, we consider the following Hamiltonian

$$
H=\sum_{i j \sigma} t_{i j} a_{i \sigma}^{+} a_{j \sigma}+\sum_{i} u_{i}-J \sum_{<i j>} \vec{S}_{i} \vec{S}_{j}
$$

where $u_{i}$ is either $u_{i}^{A}$ or $u_{i}^{M}$ depending on the ion species occupying the $i$ site:

$$
u_{i}= \begin{cases}E_{A} \sum_{\sigma} a_{i \sigma}^{+} a_{i \sigma}, & i \in A \\ E_{M} \sum_{\sigma} a_{i \sigma}^{+} a_{i \sigma}-\Delta \sum_{\sigma} a_{i \sigma}^{+} a_{i \sigma}\left(\sigma S_{i}\right), & i \in M n .\end{cases}
$$

Here $a_{i \sigma}^{+}\left(a_{i \sigma}\right)$ is the creation (annihilation) operator for a carrier with spin $\sigma$ at $i$ site; $\vec{S}_{i}$ denotes the spin of localized impurity at $i$ site ; $\Delta$ is the effective coupling constant between the localized spin and itinerant spin; $J$ is the coupling constant between the neighbouring localized impurity spins, which depends on their distance and for the AF exchange interaction case $J<0$.

By using coherent potential approximation and mean field approximation the free energy per site of the system (1) at temperature $T$ is given as [9]

$$
F(m)=E_{\text {carr }}(m)+h m x+x J \gamma m^{2} / 2-x k_{B} T \ln \left(\sum_{S^{z}= \pm S} e^{\beta(h+J \gamma m) S^{z}}\right),
$$

where $m$ is the average magnetization per lattice site, $E_{c a r r}(m)$ is the carrier energy, $h$ is the field induced by the polarization of the carrier spins, $\gamma$ is the effective number of surrounding impurities a given impurity interacts with and $\beta=1 / k_{B} T$.

By minimizing $F$ with respect to $m$ we obtain the equation for $h$, and within the Weiss molecular field theory, each impurity spin feels an effective field $\bar{h}=h+J \gamma m$, then the local magnetization is calculated by

$$
m=S B_{S}\left(\frac{\bar{h} S}{k_{B} T}\right)
$$

where $B_{S}(x)=\frac{2 S+1}{2 S} \operatorname{coth} \frac{2 S+1}{2 S} x-\frac{1}{2 S} \operatorname{coth} \frac{1}{2 S} x$ is the conventional Brillouin function and

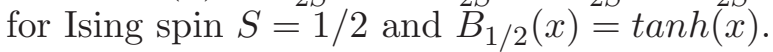

The Curie temperature is determined by differentiating both sides of Eq. (4) with respect to $m$ at $m=0$. This leads to the formula

$$
k_{B} T_{c}=\frac{S(S+1)}{3}\left(-\left.\frac{1}{x} \frac{d^{2} E_{c a r r}(m)}{d^{2} m}\right|_{m=0}+J \gamma\right) .
$$


So, we have

$$
T_{c}=T_{c 0}-T_{A F},
$$

where $T_{c 0}=-\left.\frac{S(S+1)}{3 x k_{B}} \frac{d^{2} E_{c a r r}(m)}{d^{2} m}\right|_{m=0}$ is the Curie temperature of the system in the absence of antiferromagnetic interaction between magnetic impurities; and $T_{A F}=-\frac{S(S+1)}{3 k_{B}} J \gamma$ describes the contribution of the antiferromagnetic interaction to the Curie temperature. We mention that Eq. (6), which has been derived in some early studies $[10,11]$ within the Weiss mean field theory, implies that the Curie temperature is determined by competition between the ferromagnetic and antiferromagnetic interactions. Here, the main difference between our result and that of Refs. $[10,11]$ is that we perform our calculation of $T_{c 0}$ by applying the coherent potential approximation to the coupled carrier localized spin system.

\section{NUMERICAL RESULTS AND DISCUSSION}

Our main interest is focused on the dependence of the Curie temperature on the significant model parameters, particularly, on the antiferromagnetic coupling constant $J$. Through this work we take $E_{A}$ as the origin $(=0)$ and $W$ as the unit of energy, $\gamma=6$ for simple cubic lattice. We have shown our results in figures 1-4. In Fig. 1 we have plotted Curie temperature vs. carrier density $n$ for different values of $J=-4,-8$ and $-12.10^{-4}$, for $x=0.05, E_{M}=-0.2$ and $\Delta=-0.3$. Since $T_{A F} \sim|J|$ it follows that for all $n T_{c}$ is reduced for increasing $|J|$. This constant depends on the distance between two neighbour impurities, so it depends on the impurity concentration $x$. Unfortunately, as noted in [11], non of $J$ neither $x, n, E_{M}$ of our model is directly experimentally measurable. That is why a detailed comparison between our result and experiment cannot be done. Here we choose the magnitude of $J$ in the same order as in Ref.[11]. It is seen that our $T_{c}(n)$ first increases with increasing $n$, reaches a peak and then decreases. Therefore, our CPA $T_{c}(n)$ is very different from that of the mean field approximation (MFA), where $T_{c}$ saturates for large $n$ [12]. A similar result is also obtained in other studies $[6,13]$. This difference is due to the difference in the treatment of the disorder between CPA and the MFA.

In Fig. 2, we have shown $T_{c}(n)$ for various values of $\Delta=-0.3,-0.4$ and -0.6 , for $x=0.05, E_{M}=-0.2$ and $J=-4.10^{-4}$. One can see that for almost $n, T_{c}$ increase with the magnitude of the effective coupling $\Delta$. When $|\Delta|$ is small $(|\Delta| \leq 0.4)$ the Curie temperature vanishes at a critical value $n_{c}$ larger than $x$. On the other hand, when $|\Delta|$ is large, the ferromagnetism occurs in a narrow range of $n(\leq x)$. The $T_{c}$ rises steeply and reaches a maximum at $n \approx x / 2$ and then it decreases rapidly.

Next, in Fig. 3 we have shown $T_{c}(n)$ for different impurity concentrations $x=$ $0.025,0.05$ and 0.1 , for $\Delta=-0.3, E_{M}=-0.3$ and $J=-4.10^{-4}$. The maximum $T_{c}$ is reduced for decreasing $x$. As noted in Ref.[8] it results from to the reduction of the effective bandwidth of the impurity band in the strong coupling regime, $W^{\text {eff }} \approx \sqrt{x} W$, and the maximum $T_{c 0}$ is estimated to be $\sim \sqrt{x}$ at $n \approx x / 2$. Fig. 4 displays the change of $T_{c}$ with the change of nonmagnetic potential $E_{M}$ for $x=0.05, \Delta=-0.3$ and $J=-4.10^{-4}$. In contrast to the MFA where the finite nonmagnetic potential does not affect the calculation of the Curie temperature, in CPA the negative $E_{M}$ markedly changes $T_{C}$. Comparing with the curves in Fig. 2 it is clear that $E_{M}$ simply renormalizes the effective value of $\Delta$. 


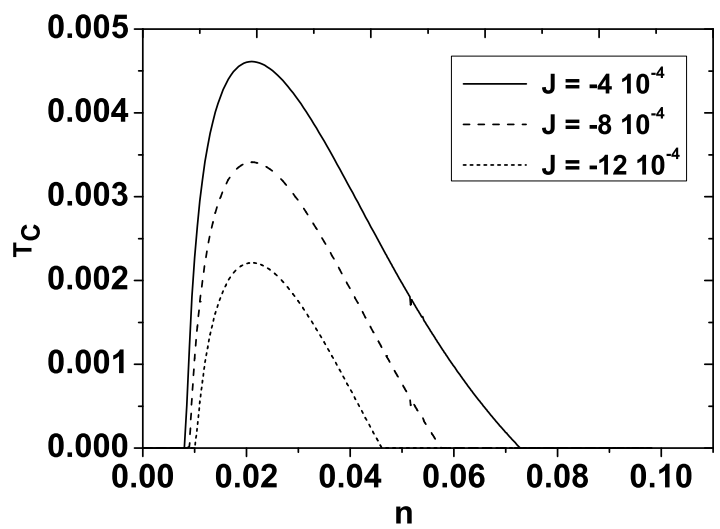

Fig. 1. Curie temperature dependent as a function of carrier density $\mathrm{n}$ for various antiferromagnetic couplings for $x=0.05, E_{M}=-0.2, \Delta=-0.3$.

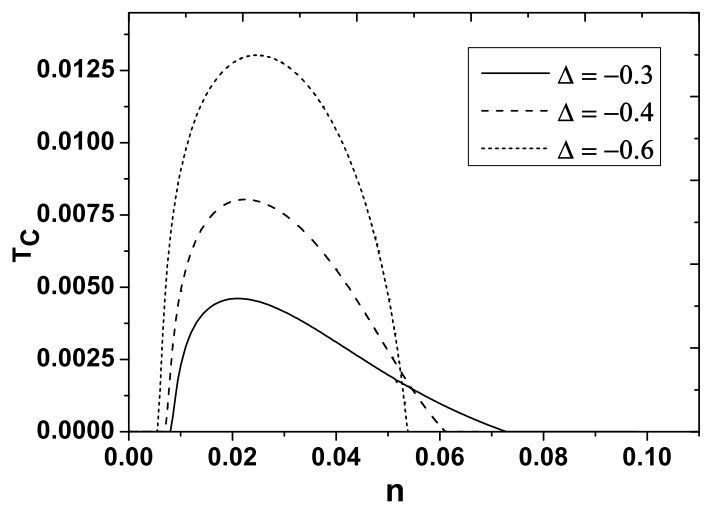

Fig. 2. Curie temperature as a function of $\mathrm{n}$ for various effective coupling constants for $x=0.05, E_{M}=-0.2, J=-4.10^{-4}$.

To summarize, we have perform a model calculation of the Curie temperature in DMS (III,Mn)V-type, where both of the exchange interaction between carrier and impurity spins, and the direct exchange interaction between magnetic impurities are taken into account, by applying the CPA and the Weiss mean-field approximation. With these methods we investigated the influence of several model parameters on $T_{c}$. We found that the Curie temperature is determined by competition between the ferromagnetic and antiferromagnetic interactions, therefore the strong antiferromagnetic superexchange interaction between nearest neighbour sites considerably reduces the Curie temperature. We showed also increasing the impurity concentration $x$ and/or the negative $E_{M}$ markedly enhances 


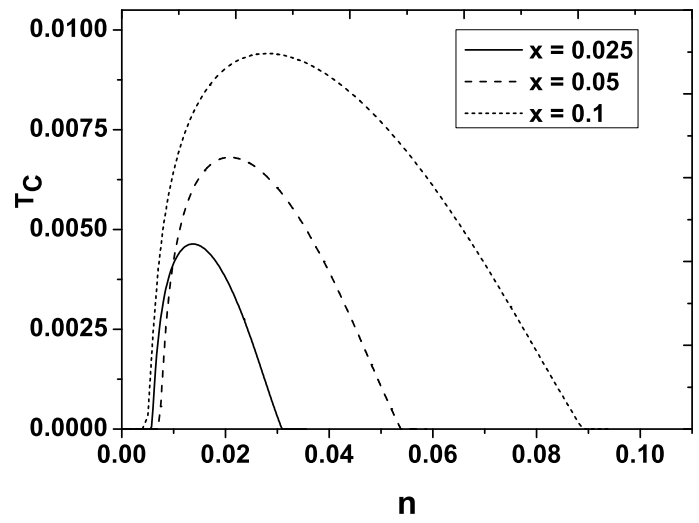

Fig. 3. Curie temperature as a function of $\mathrm{n}$ for different values of magnetic impurity concentration for $E_{M}=-0.3, \Delta=-0.3, J=-4.10^{-4}$.

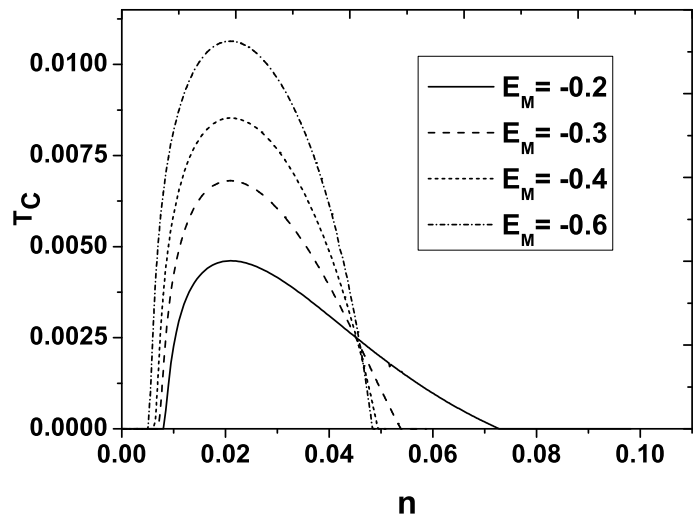

Fig. 4. Curie temperature as a function of $n$ for various nonmagnetic potential for $x=0,05, \Delta=-0.3, J=-4.10^{-4}$.

$T_{c}$. Our calculated results are in reasonable agreement with the ones obtained by a combined equation of motion/ CPA method [12].

\section{ACKNOWLEDGMENTS}

This work is supported by the National Foundation for Science and Technology Development (NAFOSTED).

\section{REFERENCES}

[1] H. Ohno, J. Magn. Magn. Mater. 200 (1999) 110.

[2] Y. Fukuma et al. , Appl. Phys. Lett. 93 (2008) 252502.

[3] R. Bouzerar and G. Bouzerar, New J. Phys. 12 (2010) 053042. 
[4] T. Dietl, H. Ohno and F. Matsukura, Phys. Rev. B63 (2001) 195205.

[5] M. Wang et al., Appl. Phys. Lett. 93 (2008) 132103.

[6] G. Bouzerar, J. Kudrnovsky, and P. Bruno, Phys. Rev. B68 (2003) 205311.

[7] S. Feng and M. Mochena, J. Phys.: Condens. Matter 18 (2006) 1441.

[8] M. Takahashi and K. Kubo, J. Phys. Soc. Jpn. 72 (2003) 2866.

[9] Vu Kim Thai, Le Duc Anh and Hoang Anh Tuan, Comm. Phys.: in press.

[10] T. Dietl et al., Science 287 (2000) 1019.

[11] S. Das Sarma, E.H. Hwang, and S. A. Kaminski, Phys. Rev. B67 (2003) 155201.

[12] M. Stier and W. Nolting, Cond-mat/1104.4222v1.

[13] M. Takahashi and K. Kubo, J. Phys. Soc. Jpn. 74 (2005) 1642.

Received 05 September 2011. 